\section{Determinantes de riesgo relacionados con la enfermedad diarreica aguda en niños me- nores de 5 años en una zona rural de Huánuco, Perú d}

Risk determinants related to acute diarrheal disease in children under 5 years of age in a rural area of Huánuco, Peru

Determinantes de risco relacionados à doença diarreica aguda em crianças menores de 5 anos em uma área rural de Huánuco, Perú

\section{iD (هulia Ortega Pacaya ${ }^{1}$ (D) Mely Ruiz-Aquino²}

\section{Resumen}

Introducción. La enfermedad diarreica aguda sigue siendo uno de los problemas de salud pública a nivel mundial que afecta principalmente a países subdesarrollados. Objetivo fue identificar los determinantes de riesgo relacionados con esta dolencia en niños menores de 5 años de la localidad de Huancapata, Ambo, Huánuco, durante el 2019. Metodología. Se realizó un estudio observacional y transversal, con diseño correlacional. La muestra fueron 101 madres de niños menores de 5 años, seleccionadas probabilísticamente. Se aplicó una guía de entrevista de las características generales, un cuestionario de determinantes de riesgo y una ficha de valoración clínica de la EDA. El análisis inferencial se realizó con la prueba de chi cuadrado, el odds ratio (OR) con sus intervalos de confianza al $95 \%$ y con un $p<0,05$. Resultados. Se halló que los determinantes hábitos de higiene: insumos inadecuados para el lavado de manos $[\mathrm{X} 2=3,631 ; \mathrm{OR}=2,2$ y $\mathrm{p}=0,046]$ y la falta de higiene de los juguetes del niño $[X 2=5,683 ; \mathrm{OR}=3,127$ y $p=0,014]$ resultaron ser estadísticamente significativos como determinantes relacionados con la EDA. Discusión. Los insumos inadecuados para el lavado de manos y la falta de lavado de los juguetes del niño, fueron los determinantes de riesgo relacionados con la EDA en los niños en estudio.

Palabras clave: Factores de riesgo, Enfermedad diarreica aguda, Niños menores de 5 años.

\section{Summary}

Introduction. Acute diarrheal disease continues to be one of the world's health problems that mainly affects underdeveloped countries. The objective is to identify the risk determinants related to this ailment in children under 5 years of age in the town
Correspondencia a:

1 Universidad de Huánuco, Huánuco, Perú

Estudiante del Programa Académico de Enfermería.

2 Universidad de Huánuco, Huánuco, Perú.

Mg. Salud Pública y Gestión Sanitaria.

Email de contacto: julia04051@@otmail.com melyruizaquino@udh.edu. pe

Procedencia y arbitraje:

No comisionado, sometido a arbitraje externo

Recibido para publicación: 30 de julio del 2021

Aceptado para publicación: 01 de septiembre del 2021

Citar como:

Ortega Pacaya J, Ruiz Aquino M. Determinantes de riesgo relacionados con la enfermedad diarreica aguda en niños menores de 5 años en una zona rural de Huánuco, Perú. Recisa UNITEPC. 2021;8(2):33-43

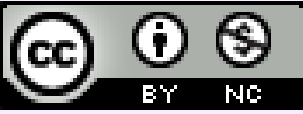

Esta obra está bajo una Licencia Creative Commons Atribución-NoComercial 4.0 Internacional. 
of Huancapata, Ambo, Huánuco, during 2019. Methodology. An observational and cross-sectional study was carried out, with a correlational design. The sample consisted of 101 mothers of children under 5 years of age, selected probabilistically. An interview guide of the general characteristics, a questionnaire of risk determinants and a clinical evaluation form of ADD were applied. The inferential analysis was performed with the chi square test, the odds ratio (OR) with its 95\% confidence intervals and with a $p<0.05$. Results. It was found that the determining hygiene habits: inadequate supplies for hand washing [X2 = 3,631; OR $=2.2$ and $p=0.046]$ and the lack of hygiene of the child's toys [X2 $=5.683$; OR $=3,127$ and $p=0.014$ ] were found to be statistically significant as determinants related to ADD. Discussion. Inadequate supplies for hand washing and lack of washing of children's toys were the risk determinants related to ADD in the children in the study.

Keywords: Risk factors, Acute diarrheal disease, Children under 5 years old.

\section{Resumo}

Introdução: A doença diarreica aguda continua sendo um dos problemas de saúde mundial que afeta, principalmente, os países subdesenvolvidos. O objetivo é identificar os determinantes de risco relacionados com esta doença em crianças menores de 5 anos da localidade de Huancapata, Ambo, Huánuco, durante o ano de 2019. Metodologia: Realizou-se um estudo observacional é transversal, com desenho correlacionar. A amostra foi de 101 mães de crianças menores de 5 anos, selecionadas probabilisticamente. Foram aplicados uma guia de entrevista das características gerais, um questionário de determinantes de risco é uma ficha de avaliação clínica da DDA. A análise inferencial foi realizada com o teste de qui quadrado, o Odds Ratio ( OR) com seus intervalos de confiança de $95 \%$ e $p<0,05$. Resultados: Constatou-se que os hábitos de higiene determinantes: insumos inadequados para a lavagem das mãos [X2=3,631; OR=2,2 ep $=0,046]$ e a falta de higiene dos brinquedos da criança [ $\mathrm{X} 2=5,683 ; \mathrm{OR}=3,127$ ep $=0,014]$, foram considerados, estatisticamente, significativos como determinantes relacionados com a DDA. Discussão: Suprimentos inadequados para a lavagem das mãos e a falta de lavagem dos brinquedos das crianças foram os determinantes de risco relacionados com a DDA nas crianças em estudo.

Palavras-chave: Fatores de risco, Doença diarreica aguda, Crianças menores de 5 anos

\section{Introducción}

Tal como señalan Ponce et al. (1) y Povea et al. (2), la enfermedad diarreica aguda (EDA), continúa siendo uno más de los problemas de salud pública a nivel mundial, afectando principalmente a los países subdesarrollados. Considerándose la población fundamentalmente vulnerable los infantes, sobre todo los lactantes, entre los cuales se evidencia alta morbilidad y mortalidad. A pesar de que los programas de control de la EDA han logrado disminuir el impacto de estas infecciones, dicha reducción no ha sido suficiente. Además, si este problema no se controla adecuadamente aumenta el riesgo de presentar complicaciones que afectan la calidad de vida de los niños (3).

Existen varios tipos clínicos de diarreas, se transmiten habitualmente por la vía fecal-oral. (4). Las medidas preventivas son ya conocidas; se evidencia tratamientos específicos $s$ basados en la rehidratación; es necesario reconocer que la EDA, es 
multifactorial, ya que no todo depende del individuo, sino también del ambiente, de las políticas públicas en salud (5), entre otros. Diversas evidencias científicas describen a las determinantes de riesgo relacionados con la EDA como: higiene doméstica deficiente, destete precoz, poco aseo personal, madre adolescente, baja escolaridad materna, procesos infecciosos recurrentes, limitado acceso al servicio de salud $(6,7)$, la carencia de saneamiento básico, el hacinamiento, la inmunización incompleta, los alimentos elaborados o almacenados en condiciones antihigiénicas (8), entre otros. Del mismo modo, Alparo et al. (9) identificaron los siguientes factores de riesgo: edad menor a 18 meses, desnutrición, uso de medicina natural, entre otros. La vacunación completa contra rotavirus es, no obstante, un factor protector frente a la diarrea con deshidratación.

Tal como explican Arévalo et al. (10), en los países en vías de desarrollo reportan un promedio de 3 episodios por niño al año en niños menores de 5 años; sin embargo, algunas áreas reportan 6 y 8 veces al año por niño. Según la Organización Mundial de la Salud (OMS) (11), la EDA es la segunda mayor causa de mortalidad de niños menores de 5 años (525 000 niños cada año; concretamente, el 2017). A nivel mundial se reportaron 1700 millones de casos de enfermedades diarreicas infantiles. En Ecuador, por ejemplo, Sánchez (12) halló una prevalencia general de EDA en el 2017 de un $24,8 \%$, con mayor proporción en niños del género masculino (55,2 \%); también hallaron determinantes familiares con mayor prevalencia de la EDA en familias medianas $(56,19 \%)$, monoparenterales $(34,29 \%)$ y funcionales $(49,52 \%)$.

A nivel del Perú, hasta la semana epidemiológica 17 del 2020 se han notificado 297. 210 episodios de EDA y 51 muertes (13). A nivel del departamento de Huánuco, en la semana epidemiológica dos del 2019 se reportaron 1284 (2,73\%) con una incidencia de 14,44 por 1000 habitantes (14). En la provincia de Ambo, localidad de Huancapata, se pudo observar que las madres no practican el lavado de manos, consumen agua sin hervir, existe hacinamiento y convivencia con los animales, entre otras determinantes de riesgo.

Este estudio se justifica porque la EDA aún sigue siendo un problema de salud pública persistente que repercute de manera negativa en el crecimiento y desarrollo saludable de los niños como población vulnerable (15); por ello, es necesario identificar precozmente las determinantes de riesgo de la EDA (16).

Por tal motivo, este estudio tuvo como objetivo de identificar los determinantes de riesgo relacionados con la EDA en niños menores de 5 años de la localidad de Huancapata, provincia de Ambo, departamento de Huánuco, Perú durante el 2019.

\section{Metodología}

Se realizó un estudio transversal y observacional de diseño fue correlacional. Este se llevó a cabo en el año 2019, en la Localidad de Huancapata, ubicado en la provincia de Ambo, situado en el sur del departamento de Huánuco, en el centro-norte del Perú.

La población fueron 137 madres con niños menores de 5 años. La muestra probabilística, seleccionada fueron 101 niños con sus respectivas madres. Se incluyeron a niños cuyas madres aceptaron firmar el consentimiento informado y niños con diagnóstico médico de la EDA durante los tres últimos meses.

Los instrumentos de recolección de datos fueron una guía de entrevista de las ca- 
racterísticas generales de la muestra en estudio, en la cual fueron consideradas las características demográficas. También se contó con una ficha de valoración de las características clínicas de la EDA y un cuestionario de determinantes de riesgo, de acuerdo a seis dimensiones: cognoscitivas, culturales, sociales, hábitos de higiene, saneamiento básico y biológicas. Este cuestionario fue validado mediante el criterio de 5 expertos y dos jueces. Asimismo, se realizó una prueba piloto a 15 madres de los niños menores de 5 años de edad, ajenos a la muestra en estudio, pero con características similares. Luego se procedió a determinar el análisis de confiabilidad a través del método KR 20 de Richardson para el cuestionario de las determinantes, donde se obtuvo un valor de 0,71 para el cuestionario de determinantes.

Previo a la recolección de datos, se solicitaron los permisos necesarios a la institución ámbito del estudio; además, se realizó una capacitación a los encuestadores para aplicar los instrumentos de medición, luego del cual se procedió a hacer las visitas domiciliarias para la aplicación de los instrumentos de recolección de datos, con el apoyo del personal de salud.

En el análisis descriptivo se usaron frecuencias absolutas y relativas. Se realizó un análisis bivariado a través de la prueba no paramétrica de chi cuadrado y se calculó el odds ratio (OR) con sus intervalos de confianza al $95 \%$; también se consideró un $p<0,05$ como significativo.

Se respetaron los principios éticos de la Declaración de Helsinski. Previa a la aplicación de los instrumentos de medición se consideró la aceptación del consentimiento informado en físico, firmado por cada responsable del niño.

\section{Resultados}

Al analizar las características demográficas de los niños menores de 5 años en estudio, respecto al grupo de edad, alrededor de la tercera parte tuvo [37,6\% (38)] de 13 a 24 meses. En cuanto al género, un $55,4 \%$ eran mujeres. Respecto a las características demográficas de las madres de los niños en estudio, el 83,2 \% de ellas eran adultas jóvenes entre 20 y 39 años. El 88,1\% procedían de la zona rural y un $50,5 \%$ radicaban en la zona del estudio por más de 3 años (ver tabla 1).

Tabla 1. Características demográficas de los niños menores de 5 años. Localidad de Huancapata Ambo - Huánuco 2019

\begin{tabular}{lll}
\hline \multirow{2}{*}{ Características demográficas del niño } & \multicolumn{2}{c}{$\mathbf{n = 1 0 1}$} \\
\cline { 3 - 4 } & $\mathbf{f i}$ & $\mathbf{\%}$ \\
\hline Grupo de edad en meses & 14 & 13,9 \\
De 1 a 12 meses & 38 & 37,6 \\
De 13 a 24 meses & 25 & 24,8 \\
De 25 a 36 meses & 21 & 20,8 \\
De 37 a 48 meses & 3 & 3,0 \\
De 49 a 60 meses & \multicolumn{2}{l}{} \\
Género & 45 & 44,6 \\
$\quad$ Masculino & 56 & 55,4 \\
$\quad$ Femenino & \multicolumn{3}{l}{} \\
\hline Características demográficas de la madre & $\mathbf{f i}$ & $\%$ \\
\hline
\end{tabular}




\section{Revista Científica de Salud UNITEPC}

\section{ISSN 2520 - 9825}

\begin{tabular}{lll}
\hline Grupo de edad en años & & \\
Adolescente (18-19 años) & 7 & 6,9 \\
Adulta joven (20-39 años) & 84 & 83,2 \\
Adulta media (40-49 años) & 10 & 9,9 \\
Zona de procedencia & & \\
$\quad$ Rural & 89 & 88,1 \\
Urbana & 11 & 10,9 \\
Urbano marginal & 1 & 1,0 \\
Tiempo de permanencia en la zona de estudio & & \\
& & \\
6 meses & 16 & 15,8 \\
1 año & 15 & 14,9 \\
2 años & 19 & 18,8 \\
Más de 3 años & 51 & 50,5 \\
\hline
\end{tabular}

En relación con las características clínicas de la EDA, se halló que un 36,6 \% (37) de los niños sí se encontraban enfermos de EDA al momento del estudio. Respecto al número de episodios de la EDA en los últimos tres meses, se evidenció que un $61,4 \%$ solo tuvo un episodio; en cuanto al número de deposiciones al día en un $43,6 \%$ eran de tres veces; en un 37,6 \% las características de las deposiciones fueron líquidas acuosas, frecuentes y abundantes; finalmente, el $24,8 \%$ de la muestra tuvo deshidratación (ver tabla 2).

Tabla 2. Descripción de las características clínicas de la Enfermedad Diarreica Aguda en niños menores de 5 años. Localidad de Huancapata, Ambo-Huánuco 2019

\begin{tabular}{|c|c|c|}
\hline \multirow{2}{*}{ Características clínicas de la EDA } & \multicolumn{2}{|c|}{$n=101$} \\
\hline & fi & $\%$ \\
\hline \multicolumn{3}{|l|}{ Presencia de la EDA en los últimos tres meses } \\
\hline Sí & 37 & 36,6 \\
\hline No & 64 & 63,4 \\
\hline \multicolumn{3}{|l|}{ Número de episodios en los últimos tres meses } \\
\hline Una vez al mes & 62 & 61,4 \\
\hline Dos veces al mes & 36 & 35,6 \\
\hline Tres veces al mes & 3 & 3,0 \\
\hline \multicolumn{3}{|l|}{ Número de deposiciones al día } \\
\hline 1 a 2 veces al día & 38 & 37,6 \\
\hline 3 veces al día & 44 & 43,6 \\
\hline Más de 3 veces al día & 19 & 18,8 \\
\hline \multicolumn{3}{|l|}{ Características de las deposiciones } \\
\hline Deposiciones líquidas acuosas frecuentes y abundantes & 38 & 37,6 \\
\hline Deposiciones sin sangre ni pus & 15 & 14,9 \\
\hline Deposiciones con sangre & 15 & 14,9 \\
\hline Otros & 14 & 13,9 \\
\hline \multicolumn{3}{|l|}{ Signos y síntomas } \\
\hline Boca, lengua, labios, piel seca y ojos hundidos & 24 & 23,8 \\
\hline
\end{tabular}




\begin{tabular}{lll} 
Deshidratación & 25 & 24,8 \\
Más de 3 deposiciones al día con sangre, moco y pus & 12 & 11,9 \\
Pérdida de apetito & 6 & 5,9 \\
Malestar general & 3 & 3,0 \\
Fiebre y vómitos & 23 & 22,8 \\
Otros & 4 & 4,0 \\
\hline
\end{tabular}

Al comparar los determinantes (cognoscitivas, culturales, sociales, saneamiento básico y biológicas) relacionados a la EDA en niños menores de 5 años, ninguna de ellas resultaron ser significativas; a diferencia de los determinantes en los hábitos de higiene, en los cuales se halló que los insumos inadecuados para el lavado de manos $\left[X^{2}=3,631 ; O R=2,2\right.$ y $\left.p=0,046\right]$ y la falta de higiene de los juguetes del niño $\left[X^{2}=5,683 ; O R=3,127\right.$ y $\left.p=0,014\right]$ resultaron ser estadísticamente significativas como determinantes relacionados a la EDA (ver tabla 3 ).

Tabla 3. Determinantes relacionados a la Enfermedad Diarreica Aguda en niños menores de 5 años. Localidad de Huancapata, Ambo - Huánuco 2019

\begin{tabular}{llclc}
\hline \multirow{2}{*}{ Determinantes cognoscitivas } & $\mathbf{n}=\mathbf{1 0 1}$ & & & \\
\cline { 2 - 5 } & $\mathbf{X}$ & OR & IC & p-valor \\
\hline Desconoce el concepto de la EDA & 0,321 & 0,565 & $(0,08-4,19)$ & 0,467 \\
Desconoce los factores de riesgo & 0,321 & 0,565 & $(0,08-4,19)$ & 0,467 \\
Desconoce las consecuencias & 0,006 & 1,047 & $(0,32-3,40)$ & 0,595 \\
Desconoce las medidas de prevención & 0,015 & 1,161 & $(0,10-13,26)$ & 0,698 \\
\hline
\end{tabular}

\begin{tabular}{|c|c|c|c|c|}
\hline \multirow{2}{*}{ Determinantes culturales } & \\
\hline & $\mathbf{X}^{2}$ & OR & IC & p-valor \\
\hline $\begin{array}{l}\text { Incumplimiento de la lactancia mater- } \\
\text { na exclusiva }\end{array}$ & 0,916 & 1,488 & $(0,66-3,36)$ & 0,227 \\
\hline Lactancia materna mixta & 2,846 & 0,399 & $(0,13-1,19)$ & 0,072 \\
\hline $\begin{array}{l}\text { Inicio inadecuado de la alimentación } \\
\text { complementaria }\end{array}$ & 0,081 & 1,140 & $(0,46-2,81)$ & 0,481 \\
\hline
\end{tabular}

\begin{tabular}{|c|c|c|c|c|}
\hline Determinantes biológicos & $\mathrm{X}^{2}$ & OR & IC & p-valor \\
\hline Vacunación incompleta & 0,260 & 0,699 & $(0,18-2,79)$ & 0,431 \\
\hline Presencia de parasitosis & 2,400 & 0,514 & $(0,22-1,20)$ & 0,092 \\
\hline Dosis incompleta de la vacuna Rota- & 2,447 & 2,526 & $(0,77-8,29)$ & 0,094 \\
\hline
\end{tabular}

virus

\begin{tabular}{|c|c|c|c|c|}
\hline Determinantes hábitos de higiene & $\overline{X^{2}}$ & OR & IC & p-valor \\
\hline $\begin{array}{l}\text { Insumos inadecuados para el lavado } \\
\text { de manos }\end{array}$ & 3,631 & 2,244 & $(0,97-5,19)$ & 0,046 \\
\hline Falta de higiene de las uñas & 1,890 & 1,847 & $(0,77-4,46)$ & 0,123 \\
\hline Falta de higiene de los juguetes del & 5,684 & 3,127 & $(1,20-8,17)$ & 0,014 \\
\hline
\end{tabular}

niño 


\begin{tabular}{|c|c|c|c|c|}
\hline $\begin{array}{l}\text { Consumo de frutas sin previa desinfec- } \\
\text { ción }\end{array}$ & 0,262 & 1,250 & $(0,53-2,94)$ & 0,385 \\
\hline Camina descalzo & 0,794 & 0,691 & $(0,31-1,56)$ & 0,247 \\
\hline Determinantes saneamiento básico & $\overline{\mathbf{X}^{2}}$ & OR & IC & p-valor \\
\hline Ausencia de agua potable & 0,103 & 0,866 & $(0,36-2,08)$ & 0,464 \\
\hline $\begin{array}{l}\text { Almacenamiento de agua en recipien- } \\
\text { tes sin tapa }\end{array}$ & 0,312 & 0,565 & $(0,08-4,19)$ & 0,467 \\
\hline Consumo de agua sin hervir & 0,000 & 1,013 & $(0,28-3,72)$ & 0,630 \\
\hline Ausencia de desagüe & 1,113 & 0,614 & $(0,25-1,53)$ & 0,204 \\
\hline $\begin{array}{l}\text { Falta de disposición de la basura en el } \\
\text { carro recolector }\end{array}$ & 0,040 & 0,916 & $(0,39-2,17)$ & 0,510 \\
\hline Determinantes sociales & $\mathrm{X}^{2}$ & OR & IC & p-valor \\
\hline $\begin{array}{l}\text { Vivienda con menos de tres habitacio- } \\
\text { nes }\end{array}$ & 1,323 & 2,500 & $(0,50-12,46)$ & 0,214 \\
\hline $\begin{array}{l}\text { Hacinamiento (más de dos personas } \\
\text { duermen en una cama) }\end{array}$ & 1,850 & 1,867 & $(0,76-4,62)$ & 0,127 \\
\hline $\begin{array}{l}\text { Crianza de animales domésticos den- } \\
\text { tro de la casa }\end{array}$ & 0,210 & 1,392 & $(0,34-5,74)$ & 0,466 \\
\hline $\begin{array}{l}\text { Ingreso económico menor al sueldo } \\
\text { básico }\end{array}$ & 0,210 & 0,718 & $(0,17-2,97)$ & 0,466 \\
\hline
\end{tabular}

\section{Discusión}

En el estudio se halló que solo los hábitos de higiene fueron los determinantes de riesgo relacionados con la EDA en niños menores de 5 años. En un primer momento se halló que los insumos inadecuados para el lavado de manos son las determinantes de riesgo relacionadas con la EDA.

Estos resultados se respaldan en las teorías del modelo del autocuidado de Orem, en las cuales se explica que la enfermería ayuda al individuo a desarrollar acciones de autocuidado para conservar la salud y la vida, recuperarse de la enfermedad o afrontar las consecuencias de dicha enfermedad (17); a su vez, el entorno es entendido en este modelo como todos aquellos factores físicos, químicos, biológicos y sociales, ya sean estos familiares o comunitarios, que influyen en la salud del individuo.

Del mismo modo, la teoría del entorno de Nightingale explica que las condiciones e influencias externas afectan a la vida (18). Además, el modelo de creencias de salud, de Rosenstock (19), describe que los comportamientos preventivos en salud, están influenciados por las percepciones individuales, los factores modificantes (amenazas percibidas) y la probabilidad de acción. También, la teoría cognoscitiva social de Bandura, explica que las personas aprenden no solamente de su propia experiencia, sino además observando las acciones de otros, y los resultados de dichas acciones proporcionarán mejoras para toda la población (20). Estos aspectos hacen referencia a que la salud del individuo esta influencia por diversas determinantes, lo que implica desarrollar un comportamiento saludable o no saludable, lo cual depende de cada 
individuo y de los diversos determinantes, estos se constituyen como factores predisponentes, precipitantes y perpetuadores del proceso salud-enfermedad.

Entre los estudios que respaldan nuestros resultados se encuentra el estudio de Aldas (21), quien halló que los padres y cuidadores no aplican las medidas higiénicas, tanto en la manipulación de alimentos, lavado de manos y esterilización del biberón. En tanto, Rodríguez et al. (22) explican que las medidas de prevención y control epidemiológico más importantes son los hábitos adecuados de higiene, como el lavado de manos con agua y jabón; además, es una forma muy económica. Al respecto, el Ministerio de Salud (MINSA) indica que el lavado de manos con agua y jabón puede eliminar hasta un $80 \%$ de los microbios causantes de enfermedades y permite la remoción mecánica de la suciedad.

Domínguez y Bravo (23) también hallaron que los factores asociados a la EDA fue la deficiencia en el lavado de manos. Al respecto, La cruz et al. (24) señalan que el saneamiento y la promoción de la higiene con participación de la comunidad pueden disminuyen la incidencia de la EDA.

En un segundo momento, se halló que la falta de higiene de los juguetes del niño es un determinante de riesgo relacionado con la EDA. Este resultado se aproxima al estudio de Myers (25), quien reportó que la transmisión del virus puede ser a través de manos o juguetes contaminados. Según Hernán (26), el 72,6 \% de madres que no lavan los juguetes del niño reportaron más episodios de la EDA. Asimismo, Granera (27) manifiesta que la mayoría de los pacientes que ingresaron a hospitalización indicaron que no realizaban el lavado de juguetes porque no les da mucho tiempo. Los juguetes son la mayoría de plástico y que muchas veces los niños están acostumbrados a llevarlos a la boca.

En tanto Lama y Castro (28) refieren que la higiene de juguetes debe ser diario, ya que estos son transmisores de la EDA; usualmente los niños se encuentran en permanente contacto con los juguetes y estos son llevados a la boca frecuentemente. Por su parte, Ochoa (29) explica que las materias fecales de los niños y los pañales sucios entran en contacto con los juguetes, convirtiéndose en un potencial de riesgo.

Los insumos inadecuados para el lavado de manos y la falta de higiene de los juguetes del niño son los determinantes de riesgo relacionados con la EDA en los niños en estudio. Frente a los hallazgos del estudio, es necesario continuar fortaleciendo las acciones de promoción de la salud y prevención de las enfermedades dirigidas a los determinantes hábitos de higiene y al tratamiento oportuno de aquellos, a fin de evitar mayores complicaciones. Además, se requiere una mayor eficiencia en las políticas públicas de salud, con enfoque de interculturalidad, para promover la prevención de la EDA en niños menores de cinco años. Asimismo, debe considerarse la necesidad de ajustar las estrategias de educación para la salud a los conceptos culturales, sociales y educativos de la población, de forma que se favorezcan las prácticas preventivas. Adicionalmente, se debe estimular el desarrollo de una cultura de salud que sea entendida como un derecho y un deber. En esa cultura, la familia debe ser responsable, individual y colectivamente, de la salud de los niños.

También, se recomienda realizar campañas integrales, articuladas bajo el enfoque de familias y viviendas saludables, que repercutan en el crecimiento y desarrollo saludable del niño. 
Los resultados del estudio no son extrapolables a otra realidad, solo son válidos para el contexto del estudio.

\section{Conflictos de interés}

Las autoras declaran no tener conflictos de interés.

\section{Referencias bibliográficas}

1. Ponce J, García M, Barriga N, Quiroz B. Morbimortalidad producida por enfermedad diarreica. Revista Científica Mundo de la Investigación y el Conocimiento. 2019 dic; 3(4): 212-232

2. Povea H. La enfermedad diarreica aguda. Rev. Cubana Pediátrica [Internet] 2019 Dic; 91(4): e928 [Consultado 2021 Ene 28] Disponible en: http://scielo.sld.cu/scielo.php?script=sci_arttext\&pid=S0034-75312019000400001\&lng=es

3. Bajaña LE. Factores de riesgos que influyen en enfermedades diarreicas agudas y su relación con la deshidratación en niños menores de 5 años, atendidos en el Centro de Salud de los Ángeles-Loreto en Quevedo, durante el segundo semestre del 2014 [Tesis de grado en Enfermería] Quevedo: Universidad Técnica Estatal de Quevedo; 2015.

4. Mamani R, Alberca A, Anne C, Cajachagua M. Estrategias para disminuir diarreas parasitosis y anemia en menores de cinco años zona altoandina Perú. Horiz. sanitario [revista en la Internet]. 2019 Dic [citado 2021 Ene 28] ;18(3):307-317.Disponible en: http://www.scielo.org.mx/scielo.php?script=sci_arttext\&pid=S2007-74592019000300307\&Ing=es. Epub 17-Ene-2020. https://doi. org/10.19136/hs.a18n3.3019.

5. Mariños C, Uchuya J, Medina J, Vidal A. Determinantes sociales de la enfermedad diarreica aguda, como identificar el riesgo y la protección para la intervención sanitaria multivalente en los niños menores de tres años en el Perú. Rev Perú epidemiol. 2014; 18(1): 1-8.

6. Hernández F, Rodríguez Z, Ferrer I, Trufero N. Enfermedades diarreicas agudas en el niño: comportamiento de algunos factores de riesgo. Rev cubana Med Gen Integr. 2018; 16(2): 129-133.

7. Piña C. Factores pronósticos de mortalidad por enfermedad diarreica aguda complicada en niños desnutridos. Panorama. Cuba y Salud. 2018 abril; 13(3): 58-64.

8. Herrera I, Fabiani N, Espejo N. Factores de riesgo para enfermedad diarreica aguda con deshidratación grave en pacientes de 2 meses a 5 años. Rev. bol. ped. [Internet] 2014; 53(2): 65-70 [Consultado 2020 Jul 22] Disponible en: http://www.scielo.org.bo/scielo.php?script=sci_arttext\&pid=S1024-06752014000200002\&lng=es

9. Alparo Herrera Indhira, Fabiani Hurtado Nelly Rocío, Espejo Herrera Nadia. Factores de riesgo para enfermedad diarreica aguda con deshidratación grave en pacientes de 2 meses a 5 años. Rev. bol. ped. [Internet]. 2014 [citado 2020 Jul 22]; 53(2): 65-70. Disponible en: http://www.scielo.org.bo/scielo.php?script=sci_arttext\&pid=S1024-06752014000200002\&Ing=es.

10. Arévalo AR, Arévalo DE, Villarroel CJ, Fernández I, Espinoza G. Enfermedad Intestinal Infecciosa (DIARREA). Rev. Méd. La Paz [Internet] 2019; 25(1): 73-85 
[Consultado 2021 Feb 05] Disponible en: http://www.scielo.org.bo/scielo.php?script=sci_arttext\&pid=S1726-89582019000100011\&lng=es

11. Enfermedades diarreicas [Internet] Organización Mundial de la Salud (OMS); 2017 [Consultado 2021 ener 21] Disponible en: https://www.who.int/es/news-room/factsheets/detail/diarrhoeal-disease

12. Sánchez NM. Factores protectores y de riesgo relacionados con las enfermedades diarreicas agudas en niños de 1 a 3 años de la Zona 7 del Ecuador [Tesis de especialidad en Medicina Familiar y Comunitaria] Loja: Universidad Nacional de Loja; 2017.

13. Centro Nacional de Epidemiologia, Prevención y Control de Enfermedades. Ministerio de Salud. Número de episodios de diarreas agudas, Perú 2015 a 2020 [Internet] [Consultado 2020 oct 2] Disponible en: https://www.dge.gob.pe/portal/docs/ vigilancia/sala/2020/SE17/edas.pdf

14. Ministerio de Salud. Semana Epidemiológica 2019 Perú [Internet] [Consultado 2019 dic 21] Disponible en: https://www.dge.gob.pe/portal/docs/vigilancia/boletines/2019/49.pdf

15. Herrera IF, Comas-García A, Mascareñas H. Impacto de las enfermedades diarreicas agudas en América Latina. Rev Latin Infect Pediatr. 2018; 31(1): 8-16

16. González EA. Aguda, prolongada y persistente en niños y su diferencia de la diarrea crónica. MEDISAN [Internet] 2017 Sep; 21(9): 2047-2060 [Consultado 2021 Feb 5] Disponible en: http://scielo.sld.cu/scielo.php?script=sci_arttext\&pi$\mathrm{d}=$ S1029-30192017000900012\&lng=es

17. Vega OM, González Escobar DS. Teoría del déficit de autocuidado: Interpretación desde los elementos conceptuales. Revista Ciencia y Cuidado. 2007; 4(4): 28-35.

18. Cano A. Florence Nightingale, la primera gran teórica de enfermería. Revista cubana de enfermería. 2004; 20(3).

19.Zárate C. Determinantes sociales de la enfermedad diarreica aguda en niños menores de 5 años en países en desarrollo [Internet] Bogotá: Universidad del Rosario; 2015 [Consultado 2021 ener 24] Disponible en: https://repository.urosario.edu. co/handle/10336/10514

20. Aprendizaje cognoscitivo social [Consultado 2020 ener 24] Disponible en: http:// biblio3.url.edu.gt/Libros/2012/Teo-Apra/4.pdf

21. Aldas AG. Estrategia para la prevención de enfermedades diarreicas aguda en niños menores de 3 años en el hospital del instituto de seguridad social del servicio de pediatría de la Ciudad de Ambato en el período septiembre 2015 - febrero 2016 [Internet] Ambato: Universidad Regional Autónoma de Los Andes (UNIANDES); 2017. Disponible en: http://dspace.uniandes.edu.ec/bitstream/123456789/6006/1/ PIUAMED031-2017.pdf

22. Rodríguez E, Arias A, Sifontes S, Luna H, Gaiti J. Epidemiología. Arch Venez Puer Ped [Internet] 2010 Mar; 73(1): 28-34 [Consultado 2021 Ene 29] Disponible en: http:// ve.scielo.org/scielo.php?script=sci_arttext\&pid=S0004-06492010000100007\&ln$g=e s$ 
23. Domínguez CL, Bravo JJ. Prevalencia y factores asociados a la enfermedad diarreica aguda en niños menores de 5 años, el Valle, 2019 [Internet] Cuenca: Universidad de Cuenca; 2019. Disponible en: https://dspace.ucuenca.edu.ec/bitstream/123456789/32645/1/PROYECTO\%20DE\%20INVESTIGACI\%C3\%93N.pdf

24. Lacruz-Rengel MA, Calderón J, Angulo F, Mata A, Quintero Y. Conocimiento materno sobre estrategias básicas de prevención en enfermedad diarreica aguda. Arch Venez Puer Ped [Internet] 2012 Dic; 75(4): 113-117 [Consultado 2021 Feb 5] Disponible en: http://ve.scielo.org/scielo.php?script=sci_arttext\&pi$\mathrm{d}=$ S0004-06492012000400005\&lng=es

25. Myers MD. Grow with us natal gastroenteritis. Rotavirus transmission and prevention. 2013; 19(2): 1-4.

26. Hernán $\mathrm{C}$. Factores socioculturales de las madres y la prevención de la enfermedad diarreica aguda en niños menores de 5 años, Yuracyacu-Chota, 2011 [Tesis de maestría en Salud Pública]. Cajamarca: Universidad Nacional de Cajamarca; 2017.

27. Granera T. Caracterización clínico-epidemiológica de diarrea aguda con sospecha por rotavirus en menores de 5 años en Hospital Alemán Nicaragüense en 2015 y 2016 [Tesis de especialidad en Pediatría]. Managua: Universidad Nacional Autónoma de Nicaragua; 2016.

28. Lama J, Mancilla L, Castro R. Enfermedades infecciosas gastrointestinales. [Tesis de licenciatura en Enfermería]. Mendoza: Universidad Nacional de Cuyo; 2014.

29. Ochoa L, Posada R, Restrepo F, Aristizábal P (AIEPI). Guía de Bolsillo [Internet] [Consultado 2020 Feb 15] Disponible en: https://www.aepap.org/sites/default/files/ aiepi.pdf 